\title{
STAKEHOLDER ENGAGEMENT IN HEALTH CARE SIMULATION
}

\author{
Sally C. Brailsford \\ Timothy Bolt \\ Con Connell \\ Jonathan H. Klein \\ Brijesh Patel \\ School of Management \\ University of Southampton \\ Southampton, SO17 1BJ, UK
}

\begin{abstract}
The RIGHT project (Research Into Global Healthcare Tools, http:/www.right.org.uk/) is a collaborative project involving five British universities, funded by the UK's Engineering and Physical Sciences Research Council. Phase 1 of the project is due to be completed in October 2009, and this paper describes one strand of the research, namely identifying some of the issues with involving stakeholders in simulation modeling in healthcare. Not the least of these is actually identifying who the stakeholders are! Other problems identified are equally tricky, as they involve deeply rooted cultural and behavioral attitudes as well as complex organizational relationships. One of the underlying aims of the next phase of RIGHT is to tackle these problems and to develop a methodology for more effective stakeholder engagement with simulation modeling.
\end{abstract}

\section{INTRODUCTION}

Simulation has been applied in the domain of healthcare for many decades. Since the 1960s, simulation models have been used worldwide to assist clinical decision-making, facility location and planning, resource allocation, evaluation of treatments, and organizational redesign. There is a massive, and constantly expanding, academic literature in this field. At the start of the 1980's, Wilson (1981) reviewed the literature on applications of simulation to healthcare. However, only 16 of the 200 papers included in the review reported recommendations that had been acted upon. More recently, Fone et al. (2004) published a systematic review of healthcare simulation models, containing 182 papers published between 1980 and 1999 , yet they too found very few examples of implementation. The authors say "... we were unable to reach any conclusions on the value of modelling in health care because the evidence of implementation was so scant." (Fone et al. 2004).

However, in most other sectors this is not the case. In manufacturing industry, for example, simulation modeling is an established part of the managerial decision-making process. It would be unthinkable for General Motors to introduce a new model of any vehicle without having used a discrete-event simulation as part of the design process for the production line. In the military, it is the same story: large simulation models are commonly used for training, strategy development or investment appraisal. The reasons for the lack of widespread adoption in healthcare are far from clear, and gaining some understanding of this problem underpinned the original thinking behind the RIGHT project (Research Into Global Healthcare Tools, <http://WWw.right.org.uk/>), under the aegis of which the work described in this paper was conducted. Because it is funded by a British Research Council, RIGHT is inevitably focused on the UK and its National Health Service (NHS), though many of the findings have global relevance. The healthcare economies of all developed countries are facing the same set of problems: spiraling organizational and delivery costs, increasing demand for services due to an ageing, educated and vocal population with high expectations, and scientific and medical advances which have made available lifeprolonging but very expensive treatments.

Part of the received wisdom in operations research in general, and simulation in particular, is that the involvement of stakeholders (who would include, in particular, 'problem owners') in the modeling process is a critical factor in successful model implementation (Robinson and Pidd 1998, Harper and Pitt 2004). In this paper we describe one strand of the RIGHT research, namely identifying the problems with involving stakeholders in simulation modeling in healthcare. Of course, we 
cannot claim to have all the answers, but we have at least clarified some of the questions, and set a research agenda for the future.

The first stage of the RIGHT project included 'rapid reviews' of the literature on applications of simulation and modeling in the three domains of healthcare, defense and commerce. Commerce covered manufacturing and service industries and all business applications. Given the vast scale of these three literatures (hundreds of thousands of papers), a conventional systematic review approach was impracticable and so a novel review methodology was adopted, similar in concept to the approach of stratified sampling. This methodology is described in Brailsford et al. (2009) and Eldabi et al. (2008). In addition, a review of stakeholder analysis tools was performed (Bolt et al. 2009). The aim was to highlight important relationships, indicate key areas of learning for the healthcare sector, and to begin to understand how success in applying simulation and modeling in these domains could potentially be transferred to healthcare.

The focus in this paper is specifically on simulation, although RIGHT does include other modeling approaches. In fact an extraordinarily wide range of methods was revealed in the healthcare modeling literature, and many of these methods were highly specific or even bespoke to the project in question, perhaps reflecting the academic imperative to publish novel methodological developments (Brailsford et al. 2009). The most widely used method was statistical analysis (a very broad catchall category) some form of which was used in $58 \%$ of the sample. Simulation, which included discrete-event, system dynamics, agent-based and Monte Carlo methods, was the next most popular approach, being used in around 30\% of the publications. Simulation methods were found to be prominent in planning and resource utilization. As part of the review, the degree of implementation of each study was rated on a three point scale: Suggested (entirely theoretical), Conceptualized (discussed with a client organization), and Implemented (actually used in practice). The proportion of papers in each category was $50 \%$ Suggested, 45\% Conceptualized and 5\% Implemented, reflecting the depressing fact that little seems to have changed since 1981.

\section{WHO ARE THE STAKEHOLDERS IN HEALTHCARE?}

The term stakeholder is defined in the management literature to mean any individual, or group of people, or organization, involved in or affected by policy making, development, implementation, or management (Freeman 1984, Brugha and Varvasovszky 2000, Schmeer 2000). Here the term policy is broadly defined to include "any national, regional, local or institutional project, programme, law, regulation or rule" (Schmeer 2000). Freeman (1984) defines a stakeholder as "any group or individual who can affect or is affected by the achievement of the firm's objectives". Clarkson (1995) extends the concept of stakeholder to "a person or groups that have, or claim, ownership, rights, or interests in a corporation and its activities, past, present, or future," thus explicitly including as legitimate stakeholders not only people within an organization who can influence its decision-making, but also those external to it who might be affected by what the organization does.

The UK's National Health Service is founded on the belief that healthcare should be "based on clinical need, not ability to pay", funded nationally through taxation and "available to all citizens of the UK free of charge at the point of delivery (NHS website, NHS Core Principles, 2009). Thus, at its broadest level, every British citizen and even every visitor to the UK is a potential or actual user of NHS services, and thus is a stakeholder in the NHS according to the above broad definitions. This is obviously not a useful approach when attempting to identify and classify the stakeholders for an actual project or policy decision. For example, when developing guidelines for scheduling patients for orthopedic surgery, the individual patients and their families, the surgeons and operating room staff, and the nurses on the orthopedic ward would be far more affected by any change to the scheduling systems than would the midwives in the delivery suite or the pharmacy staff. Clearly, for any particular problem, issue or decision, some stakeholders are more equal than others!

The management literature has plenty to say on the topic of stakeholder analysis and characterization. For example, Mitchell, Agle and Wood (1997) define the concept of salience as "the degree to which managers give priority to competing stakeholder claims" and identify three attributes which determine salience: power to influence, legitimacy of influence, and urgency of claim. A definitive stakeholder possesses all three attributes; an expectant stakeholder possesses two; a latent stakeholder possesses one; and a non-stakeholder possesses none. Their eight-way typology is dynamic, as stakeholders may shift from one class to another over time and across the issues facing the organization. It may provide a useful way of classifying healthcare stakeholders and guide to how they may best be approached, but does not bring us much closer to identifying who they are within a particular problem or intervention.

During the course of the RIGHT project, four workshops were held to which NHS staff were invited as active participants. In one such workshop held in 2007, we worked with a group of NHS staff to draw up a comprehensive list of potential stakeholders (Table 1). The goal of this was to identify the range of decision types by considering the decision-making level (policy, strategic or operational) for each identified health service stakeholder. This formed an initial basis for identifying the types of modeling issues and problems for which the stakeholder groups could be expected to have power, legitimacy, urgency and other relevance. The results of this exercise proved to serve as a useful indicator for which problem types groups would be likely to have relevant ownership, power and legitimacy. We used a simple zero-one notation to denote whether or 
not an individual or group of people could be classified as a stakeholder at this level of decision-making. Thus Parliament is clearly only involved at a policy-setting level, whereas the British Medical Association (although having a role in policymaking) is composed of individual doctors who, in their 'day jobs', will be dealing with patients at an operational level and will have to implement in practice any decisions or rulings that the BMA makes.

Another outcome of the exercise was the separation of collective organizations from their members as individuals in characterizing their stakeholder relevance. For example, patient interest groups can have an impact on policy, for example by lobbying Parliament, as well as being directly affected at the operational level, as their members are often relatives or friends of patients with a specific disease. Individual patients have very little impact on policy or strategy, and likewise very little power - unless they happen to be unlucky enough to get into the national press for being a victim of an administrative or medical error. Likewise, taxpayers as individuals have little impact (or power) even though collectively as voters they can affect Government policy.

Table 1: List of typical stakeholders and decision levels in the UK National Health Service

\begin{tabular}{|c|c|c|c|c|}
\hline & & Policy & Strategy & Operations \\
\hline Parliament & Policy committee & 1 & 0 & 0 \\
\hline \multirow[t]{3}{*}{ Government } & Health minister & 1 & 1 & 0 \\
\hline & Department of Trade \& Industry & 1 & 1 & 0 \\
\hline & Treasury & 1 & 1 & 0 \\
\hline \multirow[t]{3}{*}{ Civil services } & Social care & 1 & 1 & 0 \\
\hline & Agencies & 0 & 1 & 1 \\
\hline & Strategic Health Authorities (SHAs) & 0 & 1 & 0 \\
\hline \multirow[t]{2}{*}{ Public providers } & CEOs of NHS trusts & 0 & 1 & 1 \\
\hline & Trusts (hospitals, Primary Care Trusts) & 0 & 1 & 1 \\
\hline \multirow[t]{3}{*}{ Private providers } & Independent treatment centers & 0 & 1 & 1 \\
\hline & Private hospitals & 0 & 1 & 1 \\
\hline & Insurance companies & 0 & 1 & 1 \\
\hline \multirow[t]{8}{*}{ Professional groups } & British Medical Association & 1 & 0 & 1 \\
\hline & Royal College of Nursing & 1 & 0 & 1 \\
\hline & Allied Health Physicians & 1 & 0 & 1 \\
\hline & Royal Colleges & 1 & 1 & 0 \\
\hline & NHS Confederation & 1 & 1 & 0 \\
\hline & Educational institutions & 0 & 1 & 1 \\
\hline & Healthcare Commission & 0 & 1 & 1 \\
\hline & Allied healthcare professionals & 0 & 1 & 1 \\
\hline \multirow[t]{4}{*}{ Professionals } & General Practitioners & 0 & 1 & 1 \\
\hline & Physicians & 0 & 1 & 1 \\
\hline & Nurses & 0 & 1 & 1 \\
\hline & Surgeons & 0 & 1 & 1 \\
\hline \multirow[t]{3}{*}{ Users } & Patient interest groups & 1 & 0 & 1 \\
\hline & Patients & 0 & 0 & 1 \\
\hline & Families and informal carers & 0 & 0 & 1 \\
\hline Public & Taxpayers & 1 & 0 & 0 \\
\hline
\end{tabular}

We then tried to develop a classification scheme to map stakeholders according to their roles in a modeling project. Combining the detailed characterization of the general stakeholder literature and the relevant aspects of modeling exercises, we selected eight categories: ownership, legitimacy, power, urgency, centrality, time, money and data. While the latter three categories, time, money and data, do not normally get identified within the stakeholder classification literature, in modeling and simulation exercises these are key for planning and establishing any engagement with the stakeholders. As such it was both relevant and very useful in the modeling context to include these resource categories as well as the usual relevance cat- 
egories. Within the relevance categories, we used a simple traffic light system to depict the rating of each stakeholder: green denoted 'high', yellow 'medium' and red 'low'. Within the resource categories, the traffic lights depicted the extent to which this stakeholder could facilitate simulation modeling : green denoted 'a lot', yellow 'some' and red 'very little'.

In this classification, ownership is used as a concept reflecting a stakeholder's being responsible for a decision or being in a position to make a decision on the approach or solution to the problem being modeled. This is often a subjective perception of the stakeholder's relationship with the problem. Legitimacy is used for the extent to which a stakeholder has a legitimate right to be involved in the solution to the problem, with us taking an inclusive stance in the debate on whether such legitimate rights are based on authority, legal rights or by having 'something at risk' in the decision (Mitchell et al. 1997). Power is used by us and in the literature to reflect the influence the stakeholder may have over the situation and in affecting the outcome, irrespective of whether they have ownership or legitimate claim over it. Urgency is an indicator of the stakeholder's perceived attitude towards the importance or intensity of the problem and need to deal with it. Finally, centrality is also added to our relevancy classifications of stakeholders to reflect the actual relevance of the problem to the stakeholder's concerns or core activities. As for the three resource categories, time was basically availability to be interviewed by the modelers; money referred to the ability to allocate funding for modeling work and data reflected the stakeholder's ability to make data available in a suitable form for modeling.

We tested this schematic by classifying the stakeholders in one of the actual ongoing case studies or 'exemplars' of the RIGHT project. These exemplars were a series of practical engagements with healthcare providers to work on real problems. The particular study in question was concerned with mapping the emergency care pathways for mental health patients who self harm. This was a very complex problem, chosen because it involved a large number of different health care providers in both primary and secondary care, as well as the ambulance service, social services and even the police. One of the issues is that patients are frequently passed from one provider to another, and not only does this transfer of care occur inconsistently but also, it involves transfers of information which are not always smooth or timely. The 'system' as such is highly distributed with no clear boundaries between the various constituent parts, which can lead to communication breakdowns. Table 2 shows an extract from this classification: the actual set of identified stakeholders was much larger as it included many different staff from several hospitals and Primary Care Trusts.

Table 2: Classification of stakeholders in the self harm study to facilitate modeling

\begin{tabular}{|c|c|c|c|c|c|c|c|c|}
\hline \multirow[b]{2}{*}{ Stakeholder type } & \multicolumn{5}{|c|}{ Stakeholder Relevance Categories } & \multicolumn{3}{|c|}{ Resource Categories } \\
\hline & Ownership & Legitimacy & Power & Urgency & Centrality & Time & Money & Data \\
\hline \multicolumn{9}{|l|}{ Hospital manager } \\
\hline \multicolumn{9}{|l|}{ Hospital clinician } \\
\hline \multicolumn{9}{|l|}{ Emergency Department } \\
\hline \multicolumn{9}{|l|}{ Primary Care Trust manager } \\
\hline \multicolumn{9}{|l|}{ Primary care manager } \\
\hline \multicolumn{9}{|l|}{ General Practitioner } \\
\hline \multicolumn{9}{|l|}{ Primary-Secondary care liaison } \\
\hline \multicolumn{9}{|l|}{ Mental Heath manager } \\
\hline \multicolumn{9}{|l|}{ Mental Heath clinician } \\
\hline \multicolumn{9}{|l|}{ Ambulance Service manager } \\
\hline \multicolumn{9}{|l|}{ Ambulance clinician } \\
\hline \multicolumn{9}{|l|}{ NHS Direct manager } \\
\hline \multicolumn{9}{|l|}{ Social Services manager } \\
\hline \multicolumn{9}{|l|}{ Strategic Health Authority manager } \\
\hline \multicolumn{9}{|l|}{ Community health manager } \\
\hline \multicolumn{9}{|l|}{ Police manager } \\
\hline \multicolumn{9}{|l|}{ Local Authority manager } \\
\hline \multicolumn{9}{|l|}{ Patient proxy (mental health charity) } \\
\hline NHS Analysts & & & & & & & & \\
\hline
\end{tabular}

Key to stakeholder characteristics table: 
Note that nearly all the NHS stakeholders were coded red for time, as they are all always extremely busy. The clinicians are directly involved in the system, and thus coded green for ownership, whereas the managers were coded yellow. A few managers have access to quantitative data, and so were coded green for this, whereas clinicians have more limited access to data (and this was often qualitative or anecdotal) and so were coded yellow. The patient proxy group has no power or money, but plenty of time and high ownership. Analysts, the technical IT staff employed by the NHS mainly to do performance monitoring, have plenty of data, but limited time and no power, ownership or money.

\section{BARRIERS TO STAKEHOLDER INVOLVEMENT}

It is clear from the above discussion that the first problem is actually identifying who the key stakeholders are. This is closely related to the problem of defining the boundaries of the model. Although KISS - 'keep it simple, stupid' - is generally an excellent principle in model design, it is notoriously difficult to draw the line in a healthcare model, because to some extent, everything affects everything else. Returning to the example of modeling orthopedic surgical waiting lists, a model should probably also include (within the hospital) administrative staff, porters, the X-ray department, and maybe even the intensive care unit; and outside the hospital, general practitioners, the physiotherapy service, community nursing and rehabilitation services and possibly social services (in the case of elderly patients needing temporary residential care while they are recuperating), or employers (in the case of working-age patients unable to work while they are awaiting surgery). These are all affected by the numbers of patients undergoing orthopedic surgery. The self-harm case study was another example where it was very difficult to delineate the system boundary. However it is not sensible to attempt to model everything, and as ever the art of the simulation modeler lies in determining which elements must be included in order to make the model fit for purpose, and which elements can reasonably be ignored.

The second problem, having identified the relevant stakeholders, is gaining access to them and eliciting their views. This is far from straightforward for a number of reasons. One, NHS research ethics, will be discussed in more detail in the following section. However, even ignoring this issue, it can be difficult to involve stakeholders at all stages of any project, sometimes having to settle for subsets or 'representatives' from a wide range of potential groups (Connell et al. 1998). This is partly a factor of the complexity of any healthcare modeling project, and partly a feature of the way that simulation modeling in health has evolved as a fairly peripheral activity, not just in the UK but also worldwide. The UK Department of Health has an OR Group which has, over many years, done outstanding work. Therefore, one might reasonably expect simulation to be institutionalized and widely used across the whole NHS. Unfortunately, this is not the case. Given the size and complexity of the NHS and all the different organizations under its umbrella, there is simply too much modeling work than can be done by the Department of Health group alone. Moreover, the market economy culture introduced in the 1990's with the formation of the 'purchaser/provider split' in the NHS has led to unwillingness to share any market advantage or competitive edge gained by modeling. Many NHS organizations (Primary Care Trusts, hospitals and ambulance services) have outsourced their OR work to business consultancies, but the high prices these firms charge has meant that a lot of healthcare modeling work is also carried out as research/consultancy projects by academics. Nationally, the UK picture is highly fragmented: countless 'consultancy' projects are carried out by individual academics, published in academic journals as RIGHT and other reviews have shown, but not widely taken up and adopted elsewhere by other health providers.

A further problem is that academics and their NHS clients work to different agendas and timescales. Academics need to publish in peer-reviewed journals and must therefore demonstrate theoretical or methodological advances. This leads to complex, sophisticated models which take several years to develop, in stark contrast with the objective of the end-user: a simple, easy-to-use model (usually a spreadsheet, and ready next week!). This conflict has been aggravated by research funding allocation mechanisms such as the UK's Research Assessment Exercise. The analysis of the literature in RIGHT, plus years of experience reviewing papers for academic journals, shows that many academics are more concerned with finding an interesting test-bed to experiment on, rather than solving practical problems. This can lead to a perception by healthcare practitioners that models are complicated academic toys rather than genuinely useful and valuable tools. Cynically, one might argue that this accounts for the relative success of the big consultancies in the UK: they are better sellers and communicators, plus they charge much more, an expense which NHS managers justify to themselves by assuming they are getting a better product. It is human nature that people value what they pay highly for, and small wonder therefore that academic modelers can find it difficult to get appointments to meet busy hospital staff, whereas commercial consultancies do not always appear to have this problem.

Most of the above relates to traditional curiosity-driven academic research, where the academic has an idea which they believe could be useful to tackle some problem in the healthcare system. In this mode of research academics tend to 'push' their models out into the real world. Understandably, there are very few published examples in the academic literature of the converse 'pull' mode, whereby an academic is approached by a healthcare organization and is asked to provide genuine consultancy. Indeed, from the RIGHT research it seems clear that many successful examples of healthcare simulation modeling 
exist outside the domain of conventional journal publications. The so-called 'gray literature' - commercial and promotional material, website references and unpublished reports and presentations - contain much of interest in this field. The challenge for researchers is to find a viable means of accessing and referencing these sources, which by definition are not recorded in conventional bibliographic databases: the gray literature may be centrally important in revealing lessons to be learned from the implementation of simulation models in healthcare.

There are also cultural problems (Brailsford 2005). The healthcare industry is characterized by constant change, upheaval and stress. The traditional clinical hierarchy of doctors and nurses is being replaced by a new management hierarchy, driven in the UK at least by Government-imposed league tables and performance targets. Many healthcare workers are resistant to yet more change: they struggle just to cope with each day's workload. There is psychological resistance to methods adopted from manufacturing industry, and a feeling that such models are trying to reduce human beings to widgets in a production line and thus are doomed to failure. Moreover, in the UK the NHS has a poor track record with IT projects, one of the most famous examples being the London Ambulance Service Computer Automated Dispatch system in the early 90's (Flowers 1996).

\section{THE THORNY ISSUE OF ETHICS}

A further problem has arisen in recent years to make life more difficult for the healthcare simulation modeler - research ethics. This is written from a UK perspective, but we are aware that the same problems arise in Canada and the USA. In the UK, the NHS has always, quite rightly, had strict rules about the procedures governing medical and clinical research, rules which exist to protect patients and staff from physical and psychological harm (as well as time-wasting). However over the past five or six years these rules have extended to cover any kind of research with human participants, including any NHS staff who may be interviewed during the problem-structuring phase of a modeling project, and the use of any patient data. The NHS differentiates clearly between what it terms 'research' and 'service evaluation'. The latter does not require the approval of an NHS Research Ethics Committee, but the former does. However, there is a fine line for many healthcare simulation modelers between research and service evaluation. Academics need to call it research in order to get published! - yet often, the models they develop are aimed at service improvement, system redesign and so on. Indeed, the boundary between research and consultancy is often blurred, as healthcare modeling is all about finding practical solutions to real-world problems.

Obviously, nobody would suggest that simulation modeling should be unethical, but it does seem that the pendulum has swung too far. As anyone who has gone through the process of applying for NHS ethics approval will testify, it is a very lengthy process. It involves writing a 'protocol' - a precise and detailed specification of exactly what you intend to do, and how, and why, and with whom. Our most recent experience across a variety of health modeling projects has revealed that ethics and research governance approval can take up to 18 months or more, which is clearly a major problem if the client wants a quick answer.

The literature shows that successful, practical health modeling projects arise out of a direct need within some part of the health care system. There is a problem to be solved which drives the requirement for modeling, for example long wait times in the Emergency Department. If a business consultancy was called in to tackle this problem, they would first go through a problem structuring and information gathering process which would usually involve talking to staff - and sometimes, patients. They would probably choose to observe the system in real life. Having done all this, they would then decide which modeling technique was appropriate for this problem, and would determine any other information they might need, including specifications of the data required for their chosen approach. The modelers would then build and validate their models, develop scenarios for experimentation, document the process and make their recommendations. This is the classical 'OR modeling process' which is taught on every masters program across the world. However, if academics wished to do to the same thing, they would need to obtain NHS ethics approval before even talking to an NHS 'client'. And this is Catch 22, because in order to obtain ethical approval, it is necessary to specify in advance the exact modeling methodology which will be used; exactly who is to be interviewed, and for how long, and precisely what questions they will be asked; and exactly what data is needed!

To see why this is impossible to apply in practice, imagine Professor X has been asked to develop a model to reduce waiting times for a particular type of surgical procedure. Suppose that when she is interviewing one of the surgeons, she is told "Actually, there's another group of patients who are now supposed to get priority on this waiting list, because last month the hospital changed the clinical pathway for this diagnosis. You really need to speak to Dr Y in Cardiology, because he knows all about this." However, Professor X is not (technically) allowed to speak to Dr Y unless she had already got ethical permission to talk to someone in Cardiology! There was no way she could have predicted this six months ago when she wrote the protocol, and worse still, it could have knock-on effects. She may have originally intended to develop a queuing theory model, but because of these new priority patients, it could turn out that the structure of the real-life waiting list is now far more complicated, so that she needs to use discrete-event simulation instead. Technically this would be violating the agreed research protocol! The issue is that problem structuring - an essential part of the modeling process - is by definition open- 
ended and it is impossible to specify in advance exactly what information is needed. If one could do this, a model may not be needed at all! It is frequently said that the problem-structuring phase is the most useful part of any OR project, and people can learn more from the interactions, discussions and thinking which takes place during these initial stages than they may from the final model. Experienced consultants say that clients don't always know what their problem is - they think they do, but after preliminary discussions it can turn out to be something quite different.

It appears that there is a fundamental mismatch between clinical health research and OR health research, which is not truly reflected in the technical distinction between research and 'service evaluation'. If simulation modelers were consultants, then what they did would be pure service evaluation and there would not be a problem. However for academics, there is a research element - for example, the need to develop a new methodology to tackle a particular real-world problem, or to modify an existing technique so that it can cope with a different set of assumptions. It is interesting to speculate how many authors of those thousands of publications in the health OR literature actually obtained formal ethical approval for their studies. The key point is that simulation modeling is not like classical health services research - it is not always possible to specify a research question, a methodology and a research protocol in advance.

\section{LEARNING FROM (BITTER) EXPERIENCE}

In over twenty years of healthcare modeling, through direct personal experience as well as awareness of other modeling work presented at national and international conferences, we have seen examples of both success and failure at engaging with stakeholders. One of the most critical success factors is surely the existence of a 'champion' within the healthcare organization, a person who appreciates the value of simulation modeling even if they do not understand all the technicalities, and (crucially) who is able to persuade their colleagues that modeling is worthwhile. Building such relationships can take time but pays high dividends in the long run. A good example of this was the performance manager from the local social services department, who took a course on simulation as an elective during his MBA studies at Southampton. He was totally converted to the idea of simulation and on return to his organization instigated a series of joint projects with the university over a number of years. Without the active support of such an individual it is most unlikely that this could have happened.

It is rare to find papers in the academic literature describing failure (Connell 2001). Indeed in many of those papers in the RIGHT review which were classed as 'conceptualized', much was frequently made of the potential practical applicability of the model, even though the model had never actually been implemented. However, in academia just as in life we can learn as much, if not more, from our failures as we can from our successes. The self-harm case study described above is a good example of the problems caused by NHS ethics. The project was chosen specifically because it had so many stakeholders and would thus be particularly challenging to model. However this proved to be our downfall, because each organization had its own research ethics and separate governance procedures. The ethics approval process took so long that most of the organizations involved had restructured, the staff who were interested in the original problem (including the project champion) had all moved on to different job roles, and the initial motivation and support for the project, which was so urgent, had all but disappeared. The fundamental problem has not gone away, but has been superseded by other political imperatives. The project is currently ongoing, but the RIGHT team has had to start again to build the personal links with the relevant individuals.

Another 'learning experience' in RIGHT arose through the workshop mentioned in section 2. At this event, most of the NHS participants (around eight in number, from a range of organizations) were not told in advance with sufficient clarity what the purpose of the workshop was, and neither were most of the RIGHT researchers, a team of around ten academics. The organizers believed the event was essentially to be an unstructured sharing of ideas and problems, taking place over two days, with the aim of identifying potential projects for future collaboration. For a number of reasons, this did not work. There was a (highly influential) NHS champion, who had persuaded his colleagues to set aside two days for this workshop in the absence of a clear vision of the outcome. Some of these NHS people were clearly very skeptical, none had any idea what simulation or modeling was, and some of the more junior researchers leapt in to offer technical solutions before it was clear what the problem was. A few of the NHS participants said they were not prepared to come back on the following day, and it was not until the evening, when they were given some concrete examples of models and began to appreciate what modeling was, that they agreed to do so.

One of us has since participated in a similar event (as an observer rather than a modeler) on another project, and although in this case the outcome was more positive, it raised the same issue. This project had a steering group of around 12 people from a range of backgrounds, both NHS and academics (although not simulation modelers). The modelers began by posing the following questions: What is the problem we are trying to model? What are the boundaries? Who are the key stakeholders? What do we expect to do with the results? What are the key constraints? The NHS participants said it would be easier to answer these questions if they knew what kind of model was to be developed. It was clear that they did not mean what specific modeling approach was to be used, but rather, they had no concept of the range and potential of the models which might be used to address their problems. This is not their fault - they are skilled healthcare providers, not modelers - but is symptomatic of the challenge facing the modeling community in situations such as these. 


\section{GUIDELINES FOR SUCCESSFUL ENGAGEMENT}

Wilson (1981) identified four common factors in the 16 papers which she classed as successful implementations of simulation in health, namely: at least one author who worked at the institution concerned; timeliness (working on a high priority problem); evidence of external funding; and a detailed description of the data collection process. Twenty-three years later, Harper and Pitt (2004) listed ten factors for successful implementation:

1. Form a steering group

2. Conduct a feasibility study

3. Think carefully about level of detail and appropriate tools (OR approach required)

4. Pay attention to data quality

5. Design for wide use where possible

6. Involve end-users at all stages - build credibility

7. Maintain non-biased objective (aware of any politics)

8. Allocate necessary resources

9. Project review and foster relationship

10. Promote the results

Several of these relate to stakeholder engagement: forming a steering group, involving end-users at all stages, maintaining a non-biased and objective stance, and fostering the relationship. However although both these papers identify success factors, they do not provide practical guidance for the potential modeler on how to involve stakeholders. Mullen (1999) describes a range of methods for engaging with patients and the public. She discusses various methods for comparing preferences among diverse groups of stakeholders within the context of health policy, ranging from simple voting systems to more involved ranking systems to using gambles to pair-wise comparison methods to iterative Delphi questionnaires.

Based on our experiences with the RIGHT project and these workshops, we have become confirmed in the view that the initial meeting with stakeholders is crucial. Such meetings typically include multi-agency representation and, as well as the winning over their stakeholders, modelers need to be alert to other agendas which attendees may wish to carry forward on the back of the proposed project. We have drawn up a set of basic guidelines to assist modelers at the initial meeting with NHS stakeholders when undertaking a new project. This is based on what we have termed the 'push' mode of research, namely academics with an idea that they would like to apply to a healthcare problem. Although derived for work with the NHS, the same rules would apply in any setting.

\section{- Before the meeting}

Prepare the ground thoroughly. Setting up successful projects takes a very long time. It is essential to build as wide a set of relationships as possible. A project needs a local champion who believes in modeling. This person need not necessarily understand the technical detail of simulation, but they do need to be able to persuade their colleagues that simulation is useful. There is a trade-off here in terms of seniority: clearly it can be helpful if this person is fairly senior, as they can then use their position to influence their colleagues, but on the other hand senior colleagues are often less accessible. The research team needs at least one person who understands NHS politics and healthcare issues, and can explain issues using vocabulary which will not exclude stakeholders, but will talk their language. This need not necessarily be the person who will develop the model. As with any complex project, building trust and confidence is difficult and time-consuming. A local champion can be most helpful in recruiting others who might be definitive stakeholders; this person need not have a large involvement in the subsequent project, but can play a crucial facilitating role at the first meeting, not least in attesting (perhaps explicitly, perhaps through association) to the researchers' 'credentials'. Time invested at this stage pays off enormously later on. The modeling team need to be clear who they are going to meet, what the purpose of the meeting is, and the role each of them will play. They certainly should not approach it as a 'research meeting' or as an experiment (however it might be written up later!), but as gathering insight into the sort of problems seen as important by this group of stakeholders.

\section{- At the meeting}

Drawing a lesson from business consultancy, most successful commercial 'pitches' sell their ideas in terms of benefit to the client, not focusing on the sort of exciting, leading edge developments so beloved on academics. Pushing back the frontiers of simulation theory and practice will cut far less ice with stakeholders than the promise of the solution to a perennial or intractable problem. So the key outcome of this meeting for the modelers is to see if the tools that they have in their toolbox match up with the problems that the stakeholders appear to have. In this respect, silence is golden: modelers should listen and not talk. Stakeholders should be asked to describe their problems. The five questions posed at the end of section 5 are an excellent start: What is the problem we are trying to model? What are the boundaries? Who are the key stakeholders? What do we 
expect to do with the results? What are the key constraints? Often, this can provide an opportunity for stakeholders to gain a better understanding of how 'their' problem relates to that of other healthcare providers. The role of the modeler might be little more than occasionally prompting with questions or seeking clarification. Drawing pictures, diagrams, maps and flow charts can be helpful to clarify understanding. NEVER attempt to offer solutions in the first meeting.

Smaller groups probably work better than one large one (perhaps no more than six people in each group). The meeting should ideally take place in a comfortable large room so that people can move around, look at the computer, draw pictures on a flip-chart or whiteboard, or view a PowerPoint presentation (although this should probably be kept to a minimum).

A second key outcome of the meeting, when the modeling team will be expected to talk rather than listen, is to tell NHS staff just enough about modeling so they believe they will not be wasting their time in participating in a project. It takes skill to recognize this point, and it depends very much on the background, experience and knowledge of the audience. It is important not to dazzle NHS staff with technical scientific terms, but equally, it is vital not to patronize them. Most illustrations of what simulation is work best by using concrete examples that have a clear benefit-driven outcome, such as 'a reduction in waiting times of 18 minutes', or 'a 14\% increase in patients treated'. In our experience it is sometimes better to avoid using an example based on the specific subject area of the problem in hand, but to use an everyday example such as queuing at a supermarket checkout. This is because clinical staff in particular will inevitably spot errors in the data or model assumptions and will focus on this, rather than the basic principles the modelers are trying to convey.

\section{CONCLUSION}

The purpose of this paper has been to draw on our experiences to develop some simple practical guidelines about stakeholder engagement in simulation modeling. We recognize the challenging nature of the whole problem of stakeholder engagement and appreciate that we may have raised more questions than we have answered. The next phase of RIGHT, which at the time of writing is currently being planned, involves tackling the problem of formally identifying and classifying healthcare stakeholders, determining what different stakeholders are looking for from simulation modeling, and how their needs should best be addressed.

\section{ACKNOWLEDGEMENTS}

We are grateful for the support of the Engineering and Physical Sciences Research Council (EPSRC) under grant EP/E019900/1, and for the support and input from the other members of the RIGHT team.

\section{REFERENCES}

Brailsford, S.C.. 2005. Overcoming barriers to the implementation of OR simulation models in healthcare. The Journal of Clinical Investigative Medicine 28:312-315.

Brailsford, S.C.., Harper, P., Patel, B. and Pitt, M. 2009. An Analysis of the Academic Literature on Simulation and Modelling in Healthcare. Journal of Simulation, forthcoming.

Bolt, T., Connell, N.A.D. and Klein, J.H. 2009. Examining the Stakeholder Landscape to support Health Policy OR Projects Manuscript submitted for publication.

Brugha, R. and Varvasovszky, Z. 2000. Stakeholder analysis: a review. Health Policy and Planning. 15, 239-246

Clarkson, M.B.E. 1995. A stakeholder framework for analyzing and evaluating corporate social performance. Academy of Management Review, 20(1), pp. 92-117.

Connell, N.A.D. (2001) Evaluating soft OR: some reflections on an apparently 'unsuccessful' implementation using an SSM based approach, Journal of the Operational Research Society 52, pp. 150-160.

Connell, N.A.D., Goddard, A.R., Philp, I., and Bray, J. (1998). Patient-centred performance monitoring systems and multiagency care provision: a case study using a stakeholder participative approach. Health Services Management Research 11: pp. 92-102.

Eldabi, T., Jahangirian, M., Naseer, A., Stergioulas, L.K., Young, T. and Mustafee, N. 2008. A Survey of Simulation Techniques in Commerce and Defence. In Proceedings of the 4th Simulation Workshop 2008, Ed. S. Robinson and S.J.E. Taylor. Operational Research Society, Worcestershire, 275-284.

Flowers, S. 1996. Software Failure: Management Failure. Wiley, Chichester, UK.

Fone, D., Hollinghurst, S., Temple, M., Round, A., Lester, N., Weightman, A., Roberts, K., Coyle, E., Bevan, G. and Palmer, S. 2003. Systematic review of the use and value of computer simulation modelling in population health and health care delivery. Journal of Public Health Medicine 25:325-335. 
Freeman, R.E. 1984. Strategic Management: A Stakeholder Approach, Prentice-Hall, Englewood Cliffs, NJ.

Harper, P.R. and Pitt, M.A. 2004. On the challenges of healthcare modelling and a proposed project life-cycle for successful implementation. Journal of the Operational Research Society 55: 657-661.

Mitchell, R.K., Agle, B.R., Wood, D.J. 1997. Toward a theory of stakeholder identification and salience: defining the principles of who and what really counts. Academy of Management Review, 22:853-86.

Mullen, P. 1999. Public involvement in health care priority setting: an overview of methods for eliciting values. Health Expectations 2:222-234.

NHS website. 2009. NHS core principles. $<$ http://www.nhs.uk/nhsengland/aboutnhs/pages/NHSCorePrinciples.aspx>, accessed 24 May, 2009.

Robinson, S. and Pidd, M. 1998. Provider and Customer Expectations of Successful Simulation Projects. Journal of the Operational Research Society, 49:200-209.

Schmeer, K. (1999). Guidelines for Conducting a Stakeholder Analysis. November 1999. Bethesda, MD: Partnerships for Health Reform, Abt Associates Inc.

Wilson, J. C. T. 1981. Implementation of Computer Simulation Projects in Health Care, Journal of the Operational Research Society, 32:825-832.

Young, T., Brailsford, S., Connell, C., Davies, R., Harper, P. and Klein, J.H. 2004. Using industrial processes to improve patient care. British Medical Journal 328, 7432, 162-164

\section{AUTHOR BIOGRAPHIES}

SALLY C. BRAILSFORD is Professor of Management Science at the University of Southampton, UK. She received a BSc in Mathematics from the University of London, and MSc and PhD in Operational Research from the University of Southampton. Her research interests include simulation modeling methodologies, system dynamics, health service research and disease modeling, and the modeling of human behavior in healthcare systems. She is on the editorial boards of Health Care Management Science, the Journal of Modeling in Management and the Journal of Simulation. Email: $<$ s.c.brailsfordesoton.ac.uk $>$.

CON CONNELL is Professor of Management at the University of Southampton, UK. He has researched health-related management systems in acute and community settings for over 20 years. He has a long-standing interest in qualitative modeling of health systems using stakeholder-oriented approaches, including examination of evaluative approaches in multidisciplinary care settings. His interests in the management of knowledge in organizational settings has more recently focused on the nature of organizational stories - he is Director of the Centre for Narrative Studies at the University of Southampton. Email: <N.A.Connellesoton.ac.uk>.

JONATHAN H. KLEIN is a Senior Lecturer at the University of Southampton, UK. He has a BSc in Physics with Mathematics and an MSc in the Physics of Ion Implantation and Radiation effects in materials from the University of Sussex, and a $\mathrm{PhD}$ in Operational Research from the Royal Holloway College (University of London). His research interests include problem structuring and knowledge sharing in a variety of contexts, of which health care is one. Email: $<J \cdot H \cdot K l e i n @ s o t o n \cdot a c \cdot u k>$

TIMOTHY BOLT is a Research Fellow at the University of Southampton, UK. He received BAs from The American University and an MSc in Economics from the University of Glasgow. He earned his PhD from Waseda University in Japan with a thesis on coordinating formal and informal care services and telecare for older people. His related research interests include service improvement in care service delivery, problem structuring methods and user-requirements in care delivery. Email: <tim.boltesoton.ac.uk>.

BRIJESH PATEL is a Research Fellow at the University of Southampton, UK. He received his MBA from Gujarat University, India, and MSc and PhD in Operational Research from the University of Westminster, UK. His research interests include modeling and simulation methodologies, and analytics for performance management and strategic planning. Email:

<brijesh.patelesoton.ac.uk>. 